\title{
Protective Role of rAAV-NDI1, Serotype 5, in an Acute MPTP Mouse Parkinson's Model
}

\author{
Jennifer Barber-Singh, ${ }^{1}$ Byoung Boo Seo, ${ }^{1,2}$ Akemi Matsuno-Yagi, ${ }^{1}$ and Takao Yagi ${ }^{1}$ \\ ${ }^{1}$ Department of Molecular and Experimental Medicine, The Scripps Research Institute, 10550 North Torrey Pines Road, \\ MEM256, La Jolla, CA 92037, USA \\ ${ }^{2}$ Department of Animal Resources, College of Life \& Environmental Science, Daegu University, Jillyang, \\ Gyeongsan, Gyeongbuk 712-714, Republic of Korea
}

Correspondence should be addressed to Takao Yagi, yagi@scripps.edu

Received 15 September 2010; Accepted 22 October 2010

Academic Editor: Yuzuru Imai

Copyright (C) 2011 Jennifer Barber-Singh et al. This is an open access article distributed under the Creative Commons Attribution License, which permits unrestricted use, distribution, and reproduction in any medium, provided the original work is properly cited.

\begin{abstract}
Defects in mitochondrial proton-translocating NADH-quinone oxidoreductase (complex I) have been implicated in a number of acquired and hereditary diseases including Leigh's syndrome and more recently Parkinson's disease. A limited number of strategies have been attempted to repair the damaged complex I with little or no success. We have recently shown that the non-protonpumping, internal NADH-ubiquinone oxidoreductase (Ndi1) from Saccharomyces cerevisiae (baker's yeast) can be successfully inserted into the mitochondria of mice and rats, and the enzyme was found to be fully active. Using recombinant adenoassociated virus vectors (serotype 5) carrying our NDI1 gene, we were able to express the Ndi1 protein in the substantia nigra (SN) of C57BL/6 mice with an expression period of two months. The results show that the AAV serotype 5 was highly efficient in expressing Ndi1 in the $\mathrm{SN}$, when compared to a previous model using serotype 2, which led to nearly $100 \%$ protection when using an acute MPTP model. It is conceivable that the AAV-serotype5 carrying the NDI1 gene is a powerful tool for proof-of-concept study to demonstrate complex I defects as the causable factor in diseases of the brain.
\end{abstract}

\section{Introduction}

Parkinson's disease (PD), the second most common neurodegenerative disorder, is characterized by a loss of dopaminergic neurons in the substantia nigra $(\mathrm{SN})$ which leads to a decrease in dopamine levels and a loss of motor control. The challenge in treating PD stems from a lack of understanding with regard to what triggers the onset of the disease. Studies of the disease through human pathology or from toxin-induced models, specifically 1-methyl-4-phenyl1,2,3,6-tetrahydropyridine (MPTP), have identified three possible sources; oxidative stress, mitochondrial defects, and abnormal aggregation of proteins [1-4], and in some rare cases, inherited genetic defects. The discovery of MPTP as a neurotoxin has given neuroscientists the ability to develop animal models, mostly in mice and nonhuman primates, to investigate the mechanism(s) that may lead to PD in humans
$[5,6]$. Current evaluation strategies involve the testing of animal models through neurochemical analysis (HPLC, western blots, and immunohistochemistry) and behavioral assessment [7-9].

The use of recombinant-associated adeno virus (rAAV) has been widely explored as a gene therapy tool for the past 20 years [10]. Through extensive research, a number of serotypes have been isolated (AAV1-11) and engineered, with each showing differing selectivity and efficiency at infecting tissues ranging from the CNS (neurons) to skeletal muscles [11]. The most common serotypes that are used for gene delivery in the CNS include serotypes 2 and 5 with serotype 2 being the most widely used thus far. The goals of current gene therapy models include promoting cell survival or modification of activity in the damaged region [12]. Some of the gene therapies attempted to date include glial cell line-derived neurotrophic factor (GDNF) and enzymes 
involved in dopamine synthesis (tyrosine hydroxylase (TH) and aromatic acid decarboxylase (AADC)) with limited success $[12,13]$. More recently, a preliminary report on a clinical trial in humans was published indicating the safety of using AAV as a vehicle to introduce genes into the brain [14]. In addition, they were able to demonstrate an improvement in the patients with the use of a gene that regulates the level of GABA in the basal ganglia [14]. This initial study provides great potential for further studies and the use of other genes to modify signaling in the brain as a treatment for PD and other neurodegenerative diseases.

As mentioned above, one of the possible triggers of Parkinson's disease may involve defects in the mitochondrial respiratory chain. Therefore, our approach involves a gene therapy to complement the damaged mitochondria using the internal NADH-ubiquinone oxidoreductase derived from Saccharomyces cerevisiae (baker's yeast), NDI1 [15-20]. The NDI1 gene will be expressed in the $\mathrm{SN}$ of mice using rAAV serotype 5 , as a comparison to a previous study with this gene using rAAV serotype 2 . In addition to immunohistochemical data, behavioral testing will be used to evaluate the ability of NDI1 to protect against the toxic effects of MPTP.

\section{Materials and Methods}

2.1. Animals. Twelve-week-old male (25-30 g) C57Bl/6 mice (obtained from our in-house breeding colony) were housed four per cage in a temperature-controlled environment under 12-hr light/dark cycle with free access to food and water. The housing and treatment of the animals was conducted in accordance with the National Institutes of Health Guide for the Care and Use of Laboratory Animals, and the Institutional Animal Care and Use Committee at The Scripps Research Institute approved all procedures.

2.2. Injection of $A A V-N D I 1$. Recombinant $A A V$ serotype 5 (rAAV5) carrying the NDI1 gene (designated rAAV5NDI1) was produced by and purchased from Applied Viromics (Fremont, CA). The final viral particle concentration, estimated by dot blot assay, was determined to be $3.1 \times 10^{12}$ viral particles $/ \mathrm{ml}$. Surgical procedures were performed as previously described by Seo et al. [19]. Briefly, anesthesia was induced with $3 \%$ of isoflurane in $\mathrm{O}_{2}$, and mice were secured in a stereotaxic frame (Kopf Instruments, Tujunga, CA). Anesthesia was maintained for the duration of the surgical procedure with $1.5-2 \%$ of isoflurane in $\mathrm{O}_{2}$ through a nose tip fixed to the stereotaxic frame. All rAAV5-NDI1 injections were made using a 5$\mu \mathrm{L}$ Hamilton microsyringe with a 30 -gauge beveled needle. A single injection of $2 \mu \mathrm{L}$ rAAV5-NDI1 (suspended in PBS containing $0.1 \%$ of fluorescent beads) was made in the right hemisphere at the following coordinates (measured from bregma/dura): AP: $-3.3 \mathrm{~mm}$, ML: $1.5 \mathrm{~mm}$, and DL: $-3.9 \mathrm{~mm}$, at a rate of $0.2 \mu \mathrm{L} / \mathrm{min}$. Expression levels were verified two months after injection, and prior to any drug treatment.

2.3. MPTP Treatment. MPTP handling and safety measures were in accordance with the chemical hygiene plan developed at The Scripps Research Institute. Approximately 2 months after the rAAV5-NDI1 injection, mice were subjected to acute MPTP treatment, as reported by Seo et al. [19]. Briefly, MPTP (in sterile saline) was administered intraperitoneally at a dose of $15 \mathrm{mg} / \mathrm{kg}$ of body weight. A total of 4 injections were performed at 2-hour intervals with a $100 \%$ survival rate. The MPTP-treated groups were divided into either NDI1+MPTP $(n=14)$ or MPTP only $(n=8)$. Control animals (NDI1+Saline, $n=10$ ) were injected with the vehicle (sterile saline) in place of the MPTP solution.

2.4. Behavioral Testing. Behavioral testing was performed as described previously by Baber-Singh et al. [7]. One week prior to and 1-week post-MPTP treatment animals were tested using the elevated body swing (EBS) [7, 21, 22] and methamphetamine- (MA-) induced rotation tests. EBS and MA-induced rotation trials were videotaped and analyzed at a later time by an unbiased observer. For the EBS test, each animal was held $1 \mathrm{~cm}$ from the base of the tail and suspended approximately $1 \mathrm{~cm}$ above the table for $60 \mathrm{sec}$. Movements greater than $30^{\circ}$ from vertical were counted as a swing, and the next swing was counted only after the animal returned to or passed through the neutral position.

Prior to the administration of MA $(1.5 \mathrm{mg} / \mathrm{kg})$, animals were allowed $5 \mathrm{~min}$ to acclimate to the bowl environment. Evaluation of activity was initiated 15 min after the administration of MA, to allow for the drug to take effect, and then continuously for $40 \mathrm{~min}$. The number of quarter turns around the bowl was used to evaluate any bias created by the protection of NDI1 when challenged with MPTP or saline.

2.5. HPLC Analysis. One-half of the mice from each treatment group were euthanized for HPLC analysis of striatal DA and its metabolite levels $[19,23]$. The mice were perfused with saline, after which brains were quickly removed and frozen on dry ice, and maintained at $-80^{\circ} \mathrm{C}$ until chemical analysis was performed. The method used was similar to that outlined by Seo et al. [19]. Brains were dissected with a razor blade to approximately $2 \mathrm{~mm}$ thick sections. Striatal regions from each side of the brain were isolated separately and weighed. Each sample was homogenized by sonication in 5 volumes of ice-cold $0.2 \mathrm{M}$ perchloric acid and deproteinized by centrifugation at $14,000 \mathrm{rpm}$ for $15 \mathrm{~min}$ at $4^{\circ} \mathrm{C}$. Aromatic amines and their metabolites were separated using ionpaired reversed phase HPLC coupled with electrochemical detection (Eicom ECD-300, Kyoto, Japan). Samples $(6 \mu \mathrm{L})$ kept on ice were injected into the HPLC system equipped with an SC-3ODS column $(3 \mu \mathrm{m}, 3 \times 100 \mathrm{~mm}$; Eicom $)$ with a flow rate of $4 \mathrm{ml} / \mathrm{min}$, at room temperature. The mobile phase was composed of $0.1 \mathrm{M}$ citrate-acetate buffer, $1 \mathrm{mM}$ sodium octane sulfate, and $13 \mu \mathrm{M}$ EDTA $2 \mathrm{Na}$ with a final $\mathrm{pH}$ adjusted to 3.5 prior to adding $20 \%$ ( $\mathrm{v} / \mathrm{v}$ ) of methanol. The analytes were detected on a graphite-working electrode set at $+750 \mathrm{mV}$ versus $\mathrm{Ag} / \mathrm{AgCl}$ reference electrode. The data were collected using an EPC-500 processor (Eicom); peak 
areas were calculated using the PowerChrom software and quantified from a calibration curve of standards.

2.6. Immunohistochemistry. The remaining mice from each group were perfused with saline followed by cold $4 \%$ (w/v) paraformaldehyde solution. The brains were removed and postfixed in the paraformaldehyde solution for $1 \mathrm{hr}$ at $4^{\circ} \mathrm{C}$. Brains were frozen in OCT compound (Sakura, Torrance, $\mathrm{CA}$ ) and stored at $-20^{\circ} \mathrm{C}$ until further processing. $30-\mu \mathrm{m}$ sections were collected using a cryostat (Microm, Germany), directly mounted onto slides, and stored at $-20^{\circ} \mathrm{C}$. Immunohistochemistry using antibodies against NDI1 $(1: 250$, prepared in our laboratory), tyrosine hydroxylase (TH, 1:500, EMD Bioscience/Calbiochem, La Jolla, CA), and glial fibrillary acidic protein (GFAP, 1:250, Sigma-Aldrich, St. Louis, $\mathrm{MO}$ ) was carried out on slide sections as previously described by Seo et al. [19]. Briefly, each section was first rinsed in PBS, followed by incubation in a 3\% hydrogen peroxide solution for $30 \mathrm{~min}$ to quench native peroxidases, followed by permeabilization and blocking for nonspecific binding with $10 \%$ goat serum, $5 \%$ horse serum, and $0.1 \%$ Triton $\mathrm{X}-100 / \mathrm{PBS}$ at room temperature for $1 \mathrm{hr}$. Sections were then incubated with primary antibody overnight at $4{ }^{\circ} \mathrm{C}$. For TH and GFAP, sections were subsequently incubated with biotinylated secondary antibody for $1 \mathrm{hr}$ at room temperature followed by revelation with the $\mathrm{ABC}$ elite kit (Vector Laboratories, Burlingame, CA) and DAB (3,3'diaminobenzidine tetrachloride, Sigma-Aldrich, St. Louis, $\mathrm{MO})$. NDI1 protein staining was done using the tyramide signal amplification following the manufacturer's procedure (PerkinElmer, Boston, MA). The sections were blocked using Image-iT FX (Molecular Probes, Eugene, OR), followed by primary antibody overnight at $4^{\circ} \mathrm{C}$, and horseradish peroxidase-conjugated goat antirabbit IgG $(1: 1000$, EMD Bioscience/Calbiochem) at room temperature for $2 \mathrm{hrs}$. The sections were then rinsed 3 times in PBS for $10 \mathrm{~min}$ and then incubated with the fluorophore tyramide amplification solution $(1: 75$, dilution with amplification buffer) for $7 \mathrm{~min}$ at room temperature followed by rinsing in PBS.

2.7. NADH Activity Staining. Histochemical staining for NADH dehydrogenase activity was based on the NADHtetrazolium reductase reaction [19]. Brain sections were incubated with an $\mathrm{NADH}$-tetrazolium reductase solution (0.2 M Tris-Cl, $\mathrm{pH}$ 7.4, $1.5 \mathrm{mM} \mathrm{NADH}$, and $1.5 \mathrm{mM}$ nitro blue tetrazolium) at room temperature until sections were overstained ( $t>10 \mathrm{~min}$, bright purple), followed by removal of excess color in a series of acetone solutions $(30 \%, 60 \%$, and 90\%) for $1 \mathrm{~min}$ each, and rinsed 3 times $10 \mathrm{~min}$ in deionized water.

2.8. Western Blotting. Samples used for HPLC analysis were further processed for Western blotting using a protocol outlined in Barber-Singh et al. [7]. Samples were thoroughly mixed and neutralized with $1 \mathrm{M}$ Tris ( $\mathrm{pH} 11)$, after which $2 \mu \mathrm{L}$ of DNase $(50 \mathrm{mg} / \mathrm{ml}$, Roche, Indianapolis, IN) was added, along with a protease inhibitor cocktail (Complete Mini, Roche) containing $1 \mathrm{mM}$ EDTA. To this, SDS at a final concentration of 5\% was added, and the samples were incubated at room temperature for $1 \mathrm{hr}$ prior to protein evaluation in each sample using the Bradford method. Samples were diluted in $2 \times$ sample buffer to a final concentration of $4 \mu \mathrm{g} / \mu \mathrm{L}$. Sixty micrograms of total protein was loaded and separated on a 10\% SDS-polyacrylamide gel then transferred to a $0.22-\mu \mathrm{m}$ nitrocellulose membrane (Scleicher and Schueller, Germany). Detection was performed using the following antibodies: monoclonal mouse anti-TH 1:1000 (EMD Bioscience/Calbiochem), polyclonal rabbit anti-VMAT2 1:1000 (Chemicon, Temecula, CA), monoclonal rat-anti-DAT 1:5000 (Chemicon), monoclonal mouse anti-GAPDH 1:2000 (Chemicon), and rat-antiNDI1 1:5000 (prepared in our laboratory) [24]. Visualization of the protein bands was accomplished using the appropriate secondary, either goat antimouse horseradish peroxidase (HRP) $(1: 1000$, Pierce), antirabbit HRP $(1: 5000$, GE Healthcare, United Kingdom), or antirat HRP $(1: 10,000$, Chemicon) followed by revelation with SuperSignal West Pico chemiluminescence kit (Pierce, Rockford, IL). Chemiluminescence signals were collected on autoradiography film and the density of each band was measured using the ImageJ software [25].

2.9. Statistical Analysis. Statistical analysis was performed using the Student's $t$-test. Results are expressed as the mean \pm standard error of the mean (SEM). Statistical analysis of staining density was collected and evaluated using the ImageJ software. Threshold values were set for the entire group of subjects, for example, GFAP or TH staining and applied to all sections prior to evaluating density values. Statistical significance was set as follows, unless indicated elsewhere: $* *$ if $P<.01, * * *$ if $P<.001$.

\section{Results}

3.1. Expression of the NDI1 Protein in the Mouse SN. An important aspect of gene therapy is the ability to target the appropriate structure in vivo and to have widespread expression of protein in the desired region without interfering in the native function of surrounding regions. It is therefore crucial to verify the location and extent of NDI1 protein expression in the substantia nigra (Figures 1(a) and 1(b)). The AAV-NDI1 serotype 5 that was chosen for use in this experiment shows high levels of expression throughout the SN (Figure 1(a)), when compared to TH levels (Figure 1(b)). In addition, NADH activity staining reveals widespread NDI1 expression throughout much of the SN in serial sections, averaging $900 \mu \mathrm{m}$ in both the saline and MPTP-treated groups (Figures $2(\mathrm{a})-2(\mathrm{~d})$ ). In contrast, previous trials using AAV-NDI1 serotype 2 showed that the expression of NDI1 was predominantly limited to regions near the injection point (Figure 1(c)) when compared to TH levels (Figure 1(d)). Also, the spread (anterior-posterior) of virus throughout the SN was limited, typically $300-450 \mu \mathrm{m}$ measured by the number of NDI1-positive sections. This ranges from 10 to 15 sections depending on the placement of the injection, as the time for expression was equal 

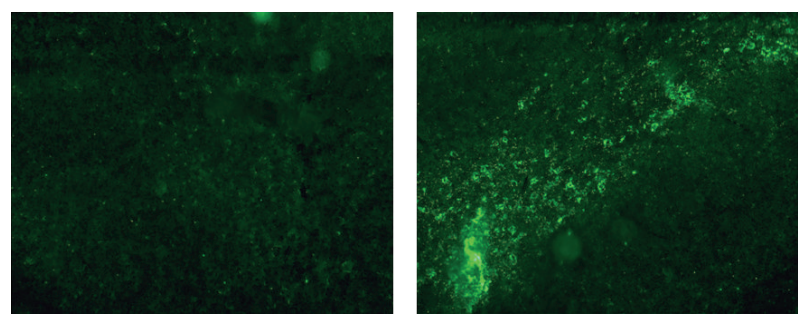

(a)
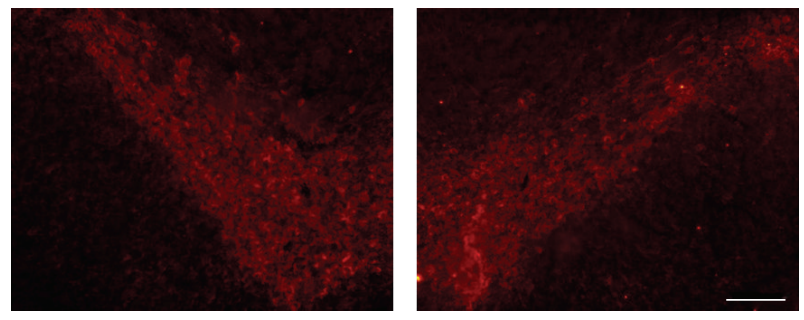

(b)
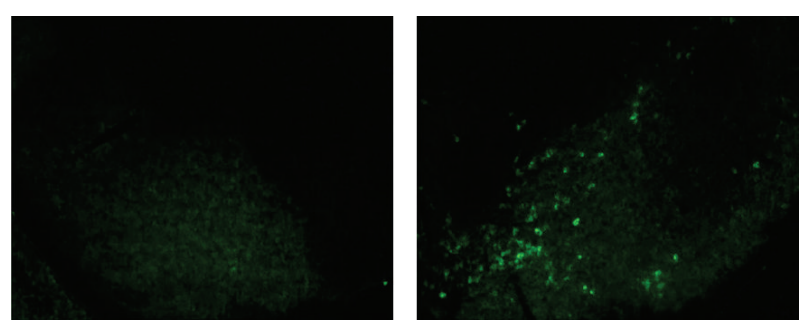

(c)
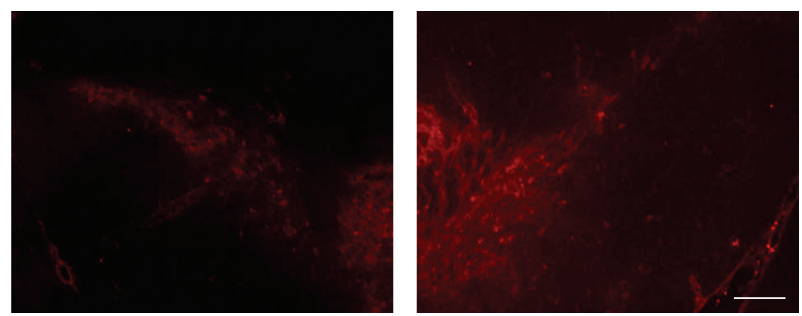

(d)

Figure 1: Comparison of NDI1 expression levels in the SN when using AAV serotype 5 versus serotype 2. All images were obtained approximately 2 months after injection of AAV-NDI1 in control mice. (a) NDI1 expression after injection of rAAV5-NDI1, (b) TH levels in SN corresponding to (a). (c) NDI1 expression after injection of AAV-NDI1 serotype 2, (d) TH levels in SN corresponding to (c). Scale bar $=200 \mu \mathrm{m}$ for (a) and (b), and $200 \mu \mathrm{m}$ for $(\mathrm{c})$ and $(\mathrm{d})$.

for both serotypes. The increase in NDI1 expression, for serotype 5 , is further established with the behavioral and immunochemical data represented below.

3.2. Effect of NDI1 Expression on Behavior Following either Saline or MPTP Treatment. The elevated body swing test (Figure 3(a)) was chosen due to the fact that it is a nondrugbased test for lateralized activity in unilateral Parkinson's models. It has been reported that the effect of administering amphetamines multiple times can lead to a sensitization resulting in poor correlation between neuronal loss and
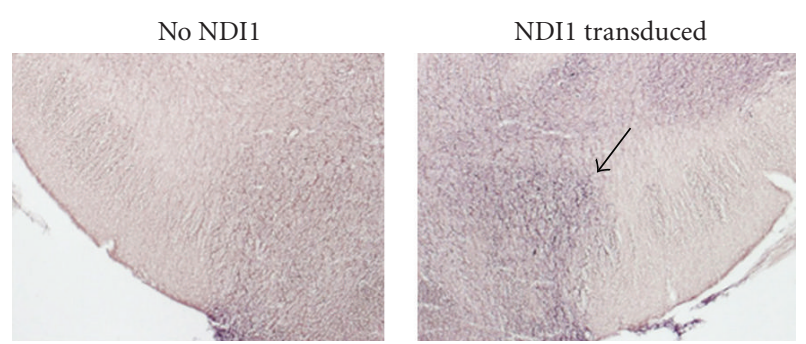

(a)
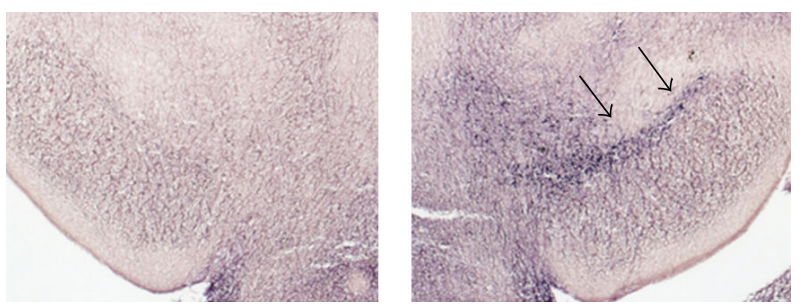

(b)
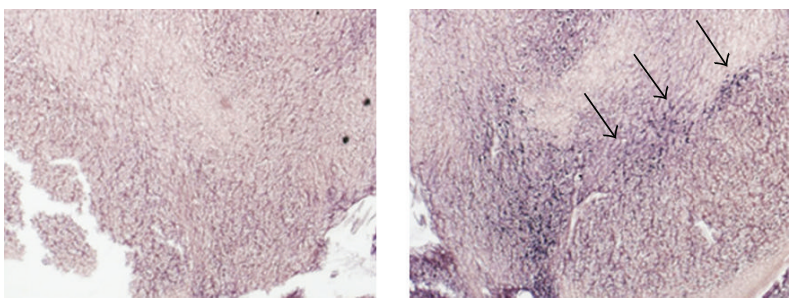

(c)
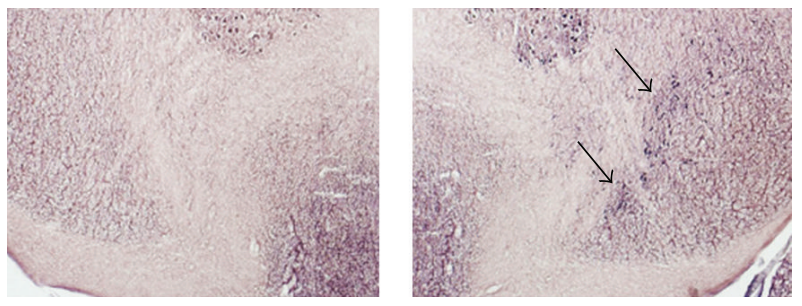

(d)

FIgure 2: Representative SN images from the NDI1 + Saline group demonstrating the spread of functional NDI1 2 months after injection. Expression of NDI1 was revealed by NADH activity staining. Image position is given relative to the injection point, which was determined by the presence of fluorescent beads added to rAAV solution prior to injection. (a) $\sim 150 \mu \mathrm{m}$ anterior of injection point, (b) point of injection, (c) $\sim 180 \mu \mathrm{m}$ posterior of (b), and (d) $\sim 720 \mu \mathrm{m}$ posterior of (b). Arrows indicate the SN.

behavioral effects. Each group of animals was first tested 1 week prior to and again approximately 1 week after MPTP treatment to evaluate the protective effect of NDI1 expression in the nigrostriatal pathway. In the pre-MPTP trials, the results confirmed no bias towards one side or the other (data not shown). The $\%$ left and \% right swings were as follows $($ mean \pm SEM $)$ : MPTP Only $(\mathrm{MO})=52.5 \%( \pm 2.6, \mathrm{~L})$ and $47.5 \%( \pm 2.6, \mathrm{R}) ; \mathrm{NDI} 1+$ Saline $(\mathrm{NS})=47.5 \%( \pm 2.8, \mathrm{~L})$ and $52.5 \%( \pm 2.8, \mathrm{R}) ; \mathrm{NDI} 1+\mathrm{MPTP}(\mathrm{NM})=48.4 \%( \pm 2.1, \mathrm{~L})$ and $51.6 \%( \pm 2.1, \mathrm{R})$. Analysis of the post-MPTP treatment 


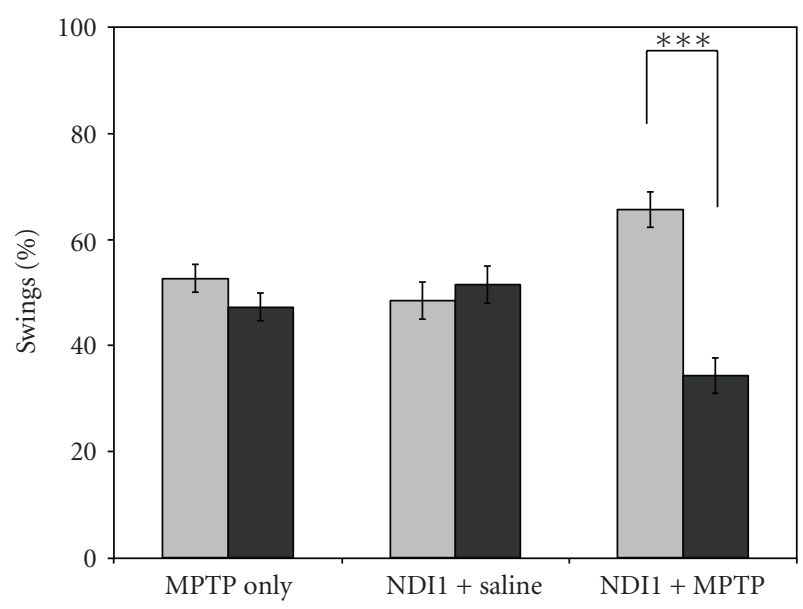

(a)

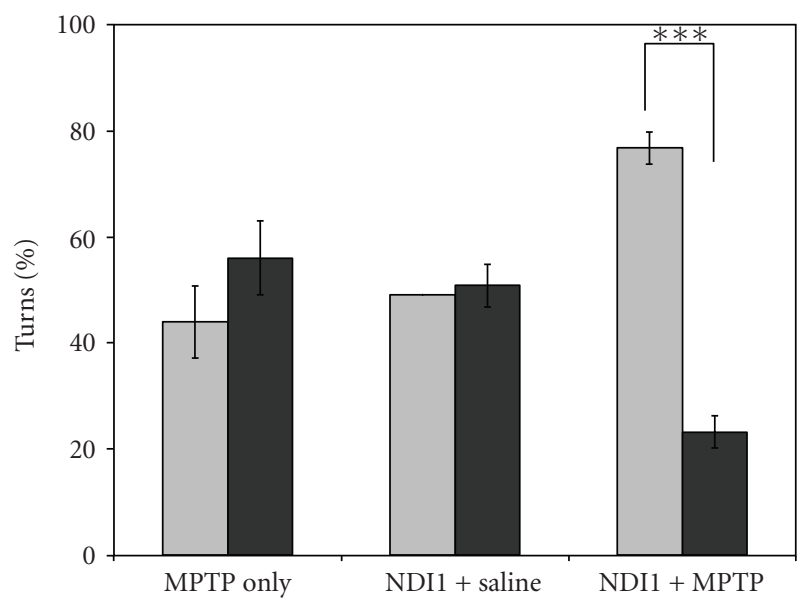

(b)

FIgUre 3: Analysis of mouse behavior tests 1 week after MPTP treatment. All trials were videotaped and analyzed at a later time by an observer blind to the identity of the animal. (a) For the EBS test, each animal was held $1 \mathrm{~cm}$ from the base of its tail and suspended approximately $1 \mathrm{~cm}$ above the table for $60 \mathrm{sec}$. The number of swings greater than $30^{\circ}$ from the vertical position was counted, and the next swing was counted only after the animal passed through vertical. (b) For the drug-induced rotation test, the number of quarter turns around the bowl and the direction were used to evaluate any bias created by the protection of NDI1 when challenged with either MPTP or saline. Data are represented as mean \pm SEM (MPTP Only, $n=8$; NDI1 + Saline, $n=10$; NDI $1+$ MPTP, $n=14)^{* * *}=P<.0005$. Light grey column, movement left; dark grey column, movement right.

revealed a significant change in behavior for the NM group only (Figure $3(\mathrm{a}))$, to $67.9 \%( \pm 3.4, \mathrm{~L})$ and $32.1 \%( \pm 3.4, \mathrm{R})$.

Methamphetamine- (MA-) induced rotation provides a robust method for evaluating unilateral damage in the Parkinson's mouse model. One week prior to and 1 week after MPTP treatment, animals were injected with $1.5 \mathrm{mg} / \mathrm{kg}$ MA. Rotational behavior (Figure 3(b)) was monitored for $40 \mathrm{~min}$, with no significant bias for the pre-MPTP treatment test (results not shown). The $\%$ turns were as follows $($ mean \pm SEM $): \mathrm{MO}=47.4 \%( \pm 7.1, \mathrm{~L})$ and $52.6 \%( \pm 7.1, \mathrm{R})$,
$\mathrm{NS}=52.4 \%( \pm 5.1, \mathrm{~L})$ and $46.6 \%( \pm 5.1, \mathrm{R})$, and $\mathrm{NM}=$ $55.0 \%( \pm 4.0, \mathrm{~L})$ and $45.0 \%( \pm 4.0, \mathrm{R})$. One week after MPTP treatment, the test was repeated (total time between trials was 3 weeks), which resulted in a significant bias for the NM group only (Figure 3(b)), 76.8\% ( $\pm 3.1, \mathrm{~L})$ and 23.2\% ( \pm 3.1 , R).

3.3. Neurochemical Analysis of the Dopaminergic System. To evaluate the level of protection that expression of NDI1 provided to the nigrostriatal system, HPLC analysis of the striatal region was used to evaluate the levels of DA, DOPAC, and serotonin (Figures 4(a)-4(c)). Significant decreases were observed for the MPTP-Only (MO) group, to approximately $30 \%$ of control (NS) for DA (Figure $4(\mathrm{a})$ ) and $75 \%$ of control for DOPAC (Figure 4(b)), as well as the nontransduced hemisphere for the NDI1-MPTP (NM), 32\% of control for DA, (Figure $4(\mathrm{a})$ ) and $73 \%$ of control for DOPAC (Figure 4(b)). For the NDI1-transduced hemisphere 100\% protection was observed for both DA (Figure 4(a)) and the major DA metabolite DOPAC (Figure 4(b)). As expected, there were no significant changes in the serotonin levels with the administration of MPTP (Figure 4(c)).

Following HPLC measurements, samples were further prepared for Western blotting analysis of the two dopaminergic transporters, VMAT2 and DAT, as well as TH and NDI1 levels after MPTP treatment (Figure 5(a)). When compared to the control group (NS), both the MO group and the nontransduced side of the NM group showed significant decreases in all proteins, with $\mathrm{TH}$ levels falling below the limit of detection. Statistical analysis of the Western blots for each protein further shows that the NDI1-transduced hemisphere provided protection against MPTP, with levels similar to that of the control group (NS) (Figure 5(b)).

3.4. Immunohistochemical Assessment of Neurodegeneration following MPTP Treatment. In order to determine functionality of the NDI1 protein, sections were incubated with $\mathrm{NADH}$ and tetrazolium as the substrates (Figure 6(a)). Darker blue staining in the $\mathrm{SN}$ and $\mathrm{CPu}$ (right hemisphere, white arrow) corresponds to the regions of functional NDI1 expression, and lighter blue/purple staining in the opposite hemisphere is presumably due to native complex I activity. Both the $\mathrm{MO}$ and the NM groups clearly have weaker NADH activity in the left hemisphere in both the SN and CPu when compared to the saline-treated group (Figure 6(a)).

In addition, to assess the degree of protection provided to the nigrostriatal system by NDI1 expression, serial striatal sections were analyzed using two immunohistochemical markers, GFAP and TH (Figure 6(b)). The GFAP staining clearly shows increased damage in the MO group, in both hemispheres, and in the nontransduced hemisphere of the NM group. Comparable results were observed for the TH staining, with a significant difference in staining between the nontransduced and NDI1-transduced hemispheres, as well as a significant decrease in $\mathrm{TH}$ staining for the $\mathrm{MO}$ group when compared to control. Statistical analysis of all tissue stained for GFAP and TH (Figures 7(a) and 7(b)) further confirms the significant differences between $\mathrm{MO}$ 


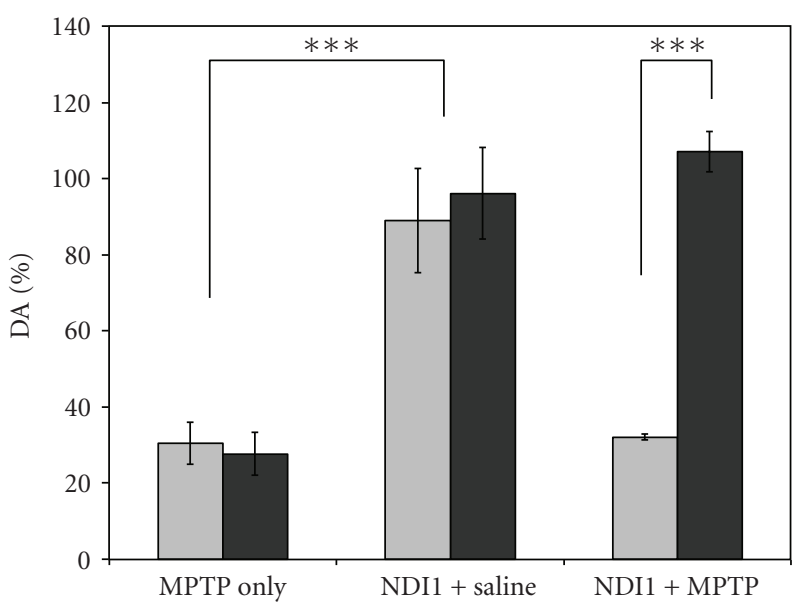

(a)

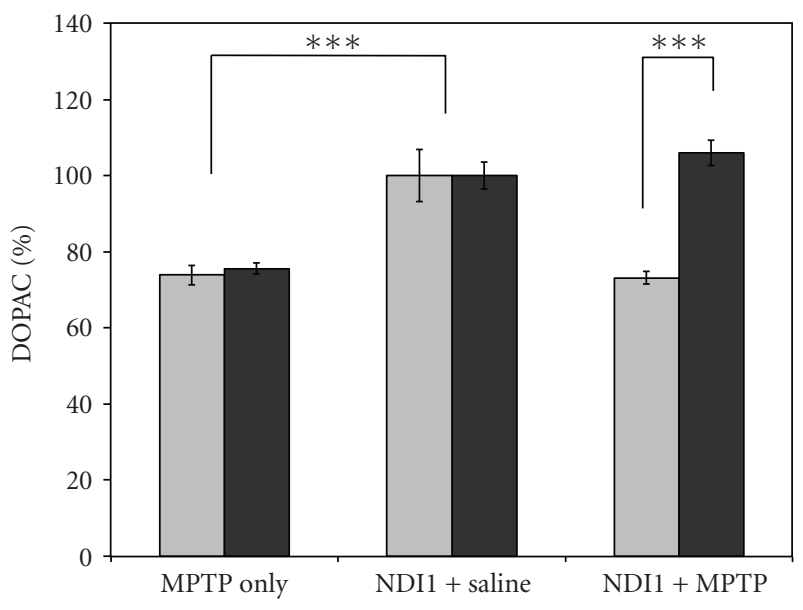

(b)

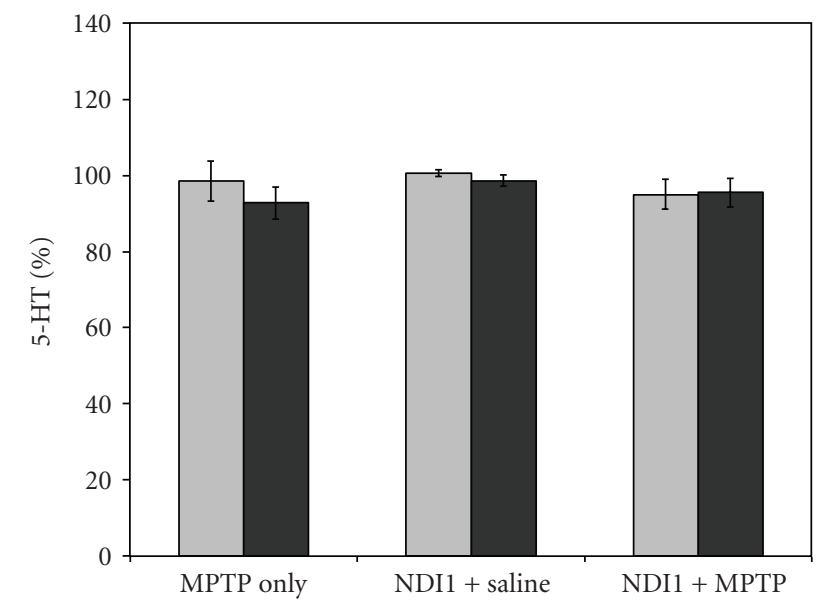

(c)

FIGURE 4: Measurement of aromatic amine levels in the mouse striatum 2 weeks after MPTP treatment. One-half of the animals from each group were euthanized, and brains were immediately frozen on dry ice prior to HPLC analysis. Striatal sections from each hemisphere were isolated separately and processed as described in the materials and methods section. (a) Dopamine (DA), (b) DOPAC, the major metabolite of DA, and (c) Serotonin (5-HT). Results are expressed as mean \pm SEM (MPTP Only, $n=5$; NDI1 + Saline, $n=5$; NDI1 + MPTP, $n=7$ ) ${ }^{* * *}=P<.0001$. Light grey column, left hemisphere; dark gray column, right hemisphere.

and NS as well as the significant difference between the hemispheres of the NM hemispheres. For the MO group, the GFAP (Figure 7(a)) staining was more than $150 \%$ of NS levels, and the nontransduced hemisphere of the NM group was also $150 \%$ of saline-treated animals. For TH staining (Figure 7(b)), MO animals had a $60 \%$ reduction in $\mathrm{TH}$ levels compared to saline-treated animals. In addition, the NM group had nearly a $90 \%$ reduction in TH staining in the nontransduced hemisphere and a slight decrease (not significant) in the TH staining in the NDI1-transduced hemisphere.

\section{Discussion}

The use of gene therapy for the treatment of Parkinson's disease has become more widespread in recent years with a focus on introducing neurotrophic factors and enzymes responsible for the production of neurotransmitters (e.g., DA and $\mathrm{GABA}$ ) as a means to prevent further loss of neurons [ 12 , $13,26]$. These remedies are most commonly introduced into the appropriate brain region using recombinant adenoassociated viruses (rAAVs). The most commonly used serotype in gene therapy applications, using $\mathrm{rAAV}$, has been type 2 which has been shown to transduce neurons in a number of brain regions, including the substantia nigra (SN) [10, 11, 26]. However, further research has produced additional recombinant serotypes that demonstrate higher transduction efficiency for specific brain regions. For example, serotype 5 was shown to be highly specific for the SN [10]. This has been confirmed with the difference in NDI1 expression levels found in the SN 2 months after injection, for serotype 2 (low) versus 5 (high). The level of expression strongly correlates with the protection provided, as evidenced through neurochemical analysis. Previous trials using rAAV2-NDI1 found $60 \%$ retention of DA levels in the striatum after acute MPTP treatment [19]. In contrast, the results here revealed 

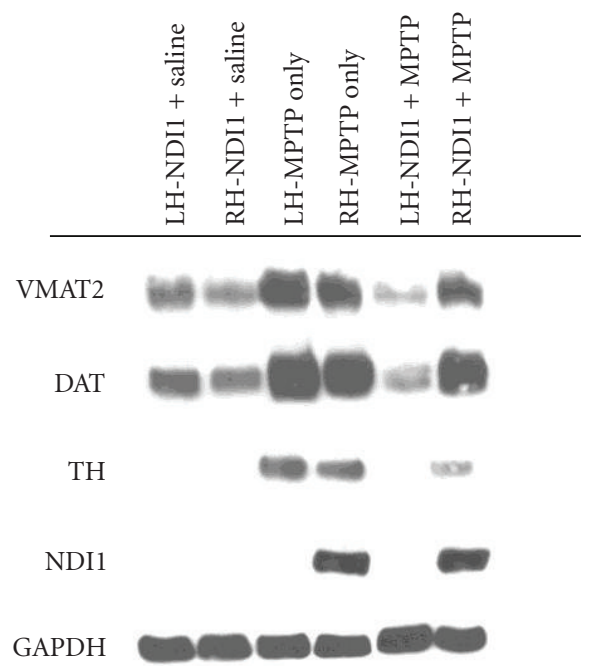

(a)
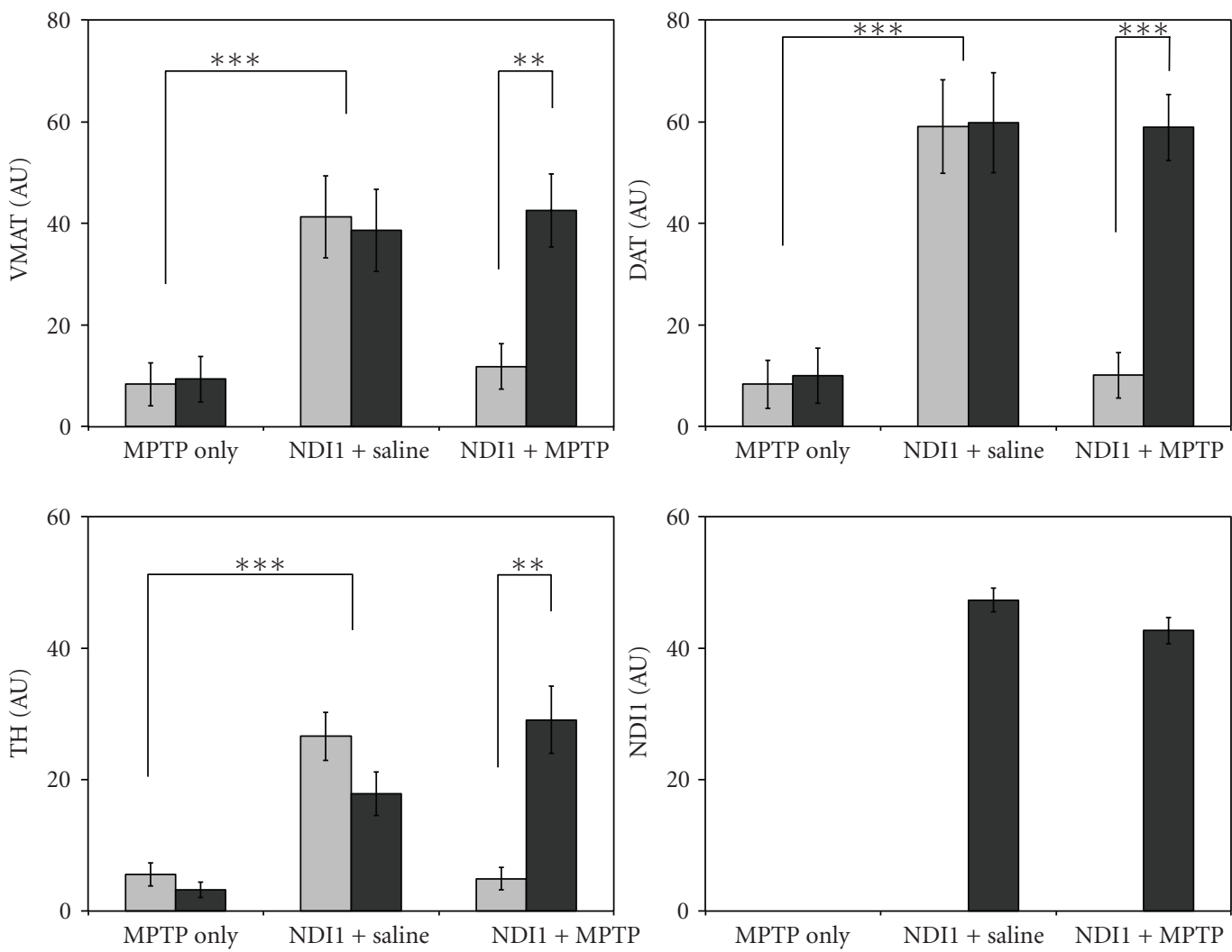

(b)

FIGURE 5: Evaluation of MPTP effects on the DAergic neurons of the striatum using mouse brain homogenates. (a) Representative Western blots, $60 \mu \mathrm{g}$ of protein per lane was loaded on a 10\% SDS-PAGE gel, (b) statistical analysis of western blots for each treatment group MPTP Only (MO), NDI1 + Saline (NS), and NDI1 + MPTP (NM), VMAT2 $(n=7,7,10)$, DAT $(n=8,8,11)$, TH $(n=5,5,7)$, and NDI1 $(n=0,9,13) .^{* *}=P<.005,{ }^{* * *}=P<.00001$. Light grey column, left hemisphere; dark grey column, right hemisphere.

that stronger and more widespread expression can result in $100 \%$ retention of DA levels in an acute MPTP model, when compared to controls.

Behavioral analysis of our MPTP model revealed lateralization of movement in the NM group only, for both the elevated body swing (EBST) and drug-induced rotation tests. For the methamphetamine-induced rotation, as expected, the animals rotated towards the lesioned side. The source of ipsilateral rotation has been described in a number of papers regarding the unilateral depletion of dopamine in the 

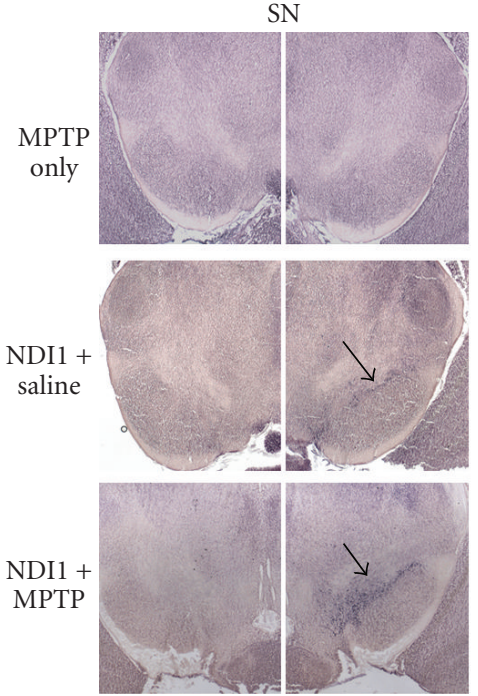

(a)
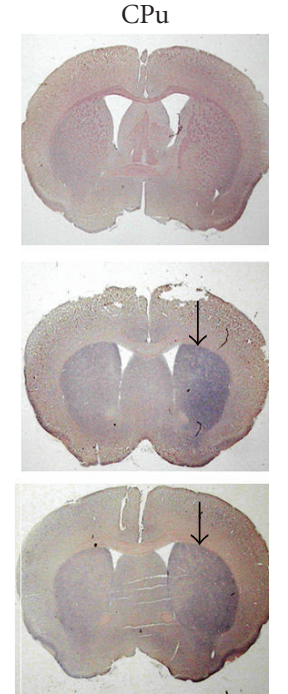
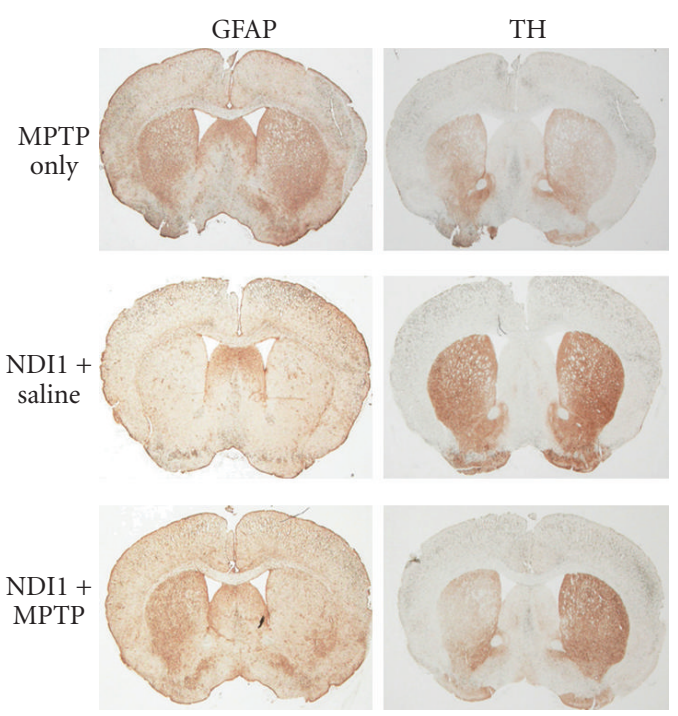

(b)

FIgure 6: Functional expression of NDI1 in the SN and CPu and protective effects following treatment with either saline or MPTP. Two weeks after the last injection, one-half of the mice were sacrificed for immunohistochemical analysis. (a) Representative NADH activity staining in the $\mathrm{SN}$ and $\mathrm{CPu}$ for each treatment group. Arrows in $\mathrm{SN}$ indicate the substantia nigra. Arrows in CPu indicate the injection side.

(b) GFAP and TH levels in CPu.

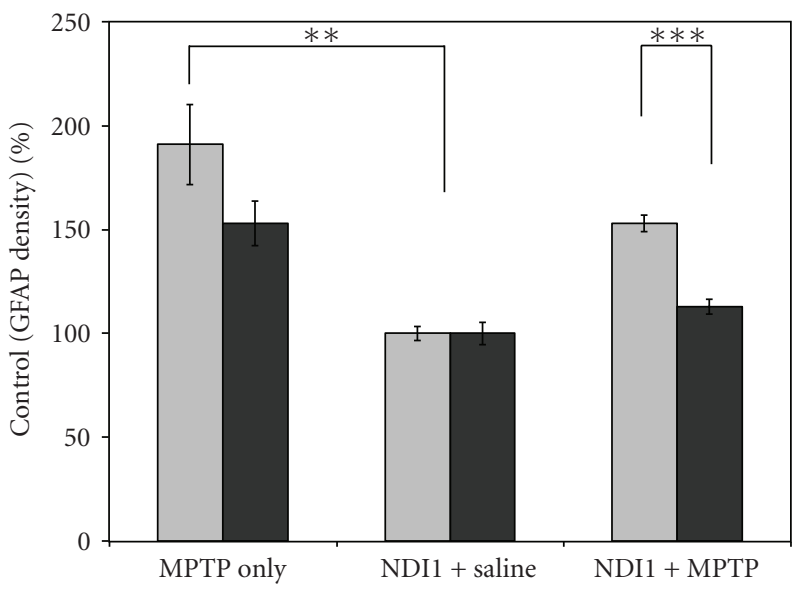

(a)

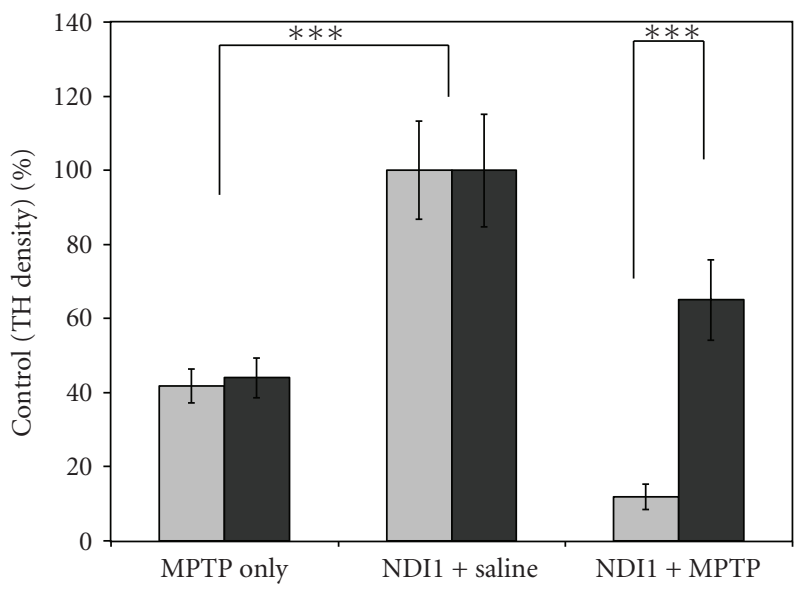

(b)

Figure 7: Statistical analysis of GFAP and TH staining density in the mouse striatum: a comparison between left and right hemisphere in MO, NS, and NM groups. Images with GFAP $(n=28)$ and TH $(n=35)$ immunostaining were collected and processed using the ImageJ software. (a) Density measurement of GFAP staining was evaluated for each hemisphere and compared to control (NDI1 + Saline, $n=10$ ), results given as $\%$ control \pm SEM (MPTP Only, $n=6$; NDI1 + MPTP, $n=12$ ). (b) The same procedure was used to evaluate TH density of each hemisphere, reported as \% control \pm SEM. (MPTP Only, $n=8$; NDI1 + Saline, $n=12 ;$ NDI1 + MPTP, $n=15$ ) ${ }^{* *}=P<.005,{ }^{* * *}=P<.0001$. Light grey column, left hemisphere; dark grey column, right hemisphere.

nigrostriatal system $[22,27,28]$. The elevated body swing test (EBS) has been used extensively with the unilateral 6-OHDA rat and mouse models $[21,22,27,29]$ and previously described for MPTP-treated mice by our group in a chronic MPTP study [7]. The results for the EBS test exhibited good correlation with the drug-induced rotation test, movement towards the lesioned side for the NM group (ipsilateral), and with a previous test in a chronic MPTP mouse model [7]. Similar tests in a 6-OHDA Parkinson model showed either a contralateral movement or no effect $[21,22,29]$. However, an interesting result published by Abrous et al. [27] demonstrated that this test may be dependent on a few factors. First, being the extent of the lesion, and second, the length of time after treatment that the test is administered; both of which may affect the changes in activity over long periods of time (i.e., months). However, in our chronic MPTP Parkinson model, we achieved the same outcome as in this experiment when testing animals more than 3 week 
after treatment [7]. And as demonstrated both in the acute and chronic models through immunohistochemical analysis, the MPTP-treated animals have extensive loss of nigrostriatal neurons, resulting in ipsilateral movement in the NDI1transduced + MPTP-treated group.

An immunohistochemical hallmark of MPTP treatment is the loss of $\mathrm{TH}$-positive neurons in both the $\mathrm{SN}$ and $\mathrm{CPu}$ $[8,30]$. This result was clearly observed in the MO group, as well as significant loss on the non-NDI1 side of the NM group. In addition, a significant increase in GFAP staining corresponded well with the loss of TH in both the $\mathrm{SN}$ and $\mathrm{CPu}$. Again, the presence of NDI1 in both the $\mathrm{SN}$ and $\mathrm{CPu}$ shows a protective effect when challenged with an acute MPTP treatment, and all immunohistochemical results were supported by the Western blot analysis. As was expected, the amount of both monoamine transporter proteins, in nonNDI1 samples, in the $\mathrm{CPu}$ was decreased when compared to controls as well as the levels of TH after 2 weeks. This marked decrease in monoamine transporters is expected as they are the primary route of $\mathrm{MPP}^{+}$sequestration and consequent toxicity in the brain [6,31-33]. In addition, once $\mathrm{MPP}^{+}$has entered the neuron, there is a passive transport into the mitochondria resulting in inhibition of complex I activity and possibly the overproduction of reactive oxygen species (ROS) [3, 34-37]. The presence of NDI1 in the NM group has prevented the deleterious effects of $\mathrm{MPP}^{+}$in the neuron which resulted in transporter levels that were not significantly altered compared to controls and only a slight decrease in TH levels.

\section{Conclusion}

In conclusion, all results obtained demonstrate a clear protective effect of NDI1 in the dopaminergic system. The use of serotype 5 in the dopaminergic neurons resulted in greater expression efficiency and consequently better protection when challenged with MPTP in an acute PD mouse model. The use of behavioral testing in conjunction with neurochemical analysis provided a more complete evaluation of the unilateral MPTP PD model. These results provide further support for the use of NDI1 as a gene therapy for the treatment of PD and the possibility for use in other mitochondrial complex I-deficient diseases.

\section{Acknowledgments}

The authors would like to thank Drs. Eiko NakamaruOgiso and Mathieu Marella (The Scripps Research Institute) for their discussion and assistance. This work was partly supported by NIH Grant no. R01DK053244.

\section{References}

[1] P. M. Abou-Sleiman, M. M. K. Muqit, and N. W. Wood, "Expanding insights of mitochondrial dysfunction in Parkinson's disease," Nature Reviews Neuroscience, vol. 7, no. 3, pp. 207-219, 2006.

[2] M. R. Cookson, "The biochemistry of Parkinson's disease," Annual Review of Biochemistry, vol. 74, pp. 29-52, 2005.
[3] W. Dauer and S. Przedborski, "Parkinson's disease: mechanisms and models," Neuron, vol. 39, no. 6, pp. 889-909, 2003.

[4] A. H. V. Schapira, "Etiology of Parkinson's disease," Neurology, vol. 66, no. 10, supplement 4, pp. S10-S15, 2006.

[5] T. R. Flotte, "Gene therapy: the first two decades and the current state-of-the-art," Journal of Cellular Physiology, vol. 213, no. 2, pp. 301-305, 2007.

[6] S. G. Speciale, "MPTP: insights into parkinsonian neurodegeneration," Neurotoxicology and Teratology, vol. 24, no. 5, pp. 607-620, 2002.

[7] J. Barber-Singh, B. B. Seo, E. Nakamaru-Ogiso, Y. S. Lau, A. Matsuno-Yagi, and T. Yagi, "Neuroprotective effect of longterm NDI1 gene expression in a chronic mouse model of parkinson disorder," Rejuvenation Research, vol. 12, no. 4, pp. 259-267, 2009.

[8] R. Kurosaki, Y. Muramatsu, H. Kato, and T. Araki, "Biochemical, behavioral and immunohistochemical alterations in MPTP-treated mouse model of Parkinson's disease," Pharmacology Biochemistry and Behavior, vol. 78, no. 1, pp. 143-153, 2004.

[9] M. Sedelis, R. K. W. Schwarting, and J. P. Huston, "Behavioral phenotyping of the MPTP mouse model of Parkinson's disease," Behavioural Brain Research, vol. 125, no. 1-2, pp. 109125, 2001.

[10] C. Burger, K. Nash, and R. J. Mandel, "Recombinant adenoassociated viral vectors in the nervous system," Human Gene Therapy, vol. 16, no. 7, pp. 781-791, 2005.

[11] Z. Wu, A. Asokan, and R. J. Samulski, "Adeno-associated virus serotypes: vector toolkit for human gene therapy," Molecular Therapy, vol. 14, no. 3, pp. 316-327, 2006.

[12] E. A. Burton, J. C. Glorioso, and D. J. Fink, "Gene therapy progress and prospects: Parkinson's disease," Gene Therapy, vol. 10, no. 20, pp. 1721-1727, 2003.

[13] M. J. During and P. Leone, "Targets for gene therapy of Parkinson's disease: growth factors, signal transduction, and promoters," Experimental Neurology, vol. 144, no. 1, pp. 7481, 1997.

[14] M. G. Kaplitt, A. Feigin, C. Tang et al., "Safety and tolerability of gene therapy with an adeno-associated virus (AAV) borne GAD gene for Parkinson's disease: an open label, phase I trial," Lancet, vol. 369, no. 9579, pp. 2097-2105, 2007.

[15] B. B. Seo, T. Kitajima-Ihara, E. K. L. Chan, I. E. Scheffler, A. Matsuno-Yagi, and T. Yagi, "Molecular remedy of complex I defects: rotenone-insensitive internal $\mathrm{NADH}$-quinone oxidoreductase of Saccharomyces cerevisiae mitochondria restores the NADH oxidase activity of complex I-deficient mammalian cells," Proceedings of the National Academy of Sciences of the United States of America, vol. 95, no. 16, pp. 9167-9171, 1998.

[16] T. Yagi, B. B. Seo, E. Nakamaru-Ogiso et al., "Can a single subunit yeast NADH dehydrogenase (Ndi1) remedy diseases caused by respiratory complex I defects?" Rejuvenation Research, vol. 9, no. 2, pp. 191-197, 2006.

[17] M. Marella, B. B. Seo, T. Yagi, and A. Matsuno-Yagi, "Parkinson's disease and mitochondrial complex I: a perspective on the Ndil therapy," Journal of Bioenergetics and Biomembranes, vol. 41, no. 6, pp. 493-497, 2009.

[18] B. B. Seo, E. Nakamaru-Ogiso, P. Cruz, T. R. Flotte, T. Yagi, and A. Matsuno-Yagi, "Functional expression of the single subunit $\mathrm{NADH}$ dehydrogenase in mitochondria in vivo: a potential therapy for complex I deficiencies," Human Gene Therapy, vol. 15, no. 9, pp. 887-895, 2004.

[19] B. B. Seo, E. Nakamaru-Ogiso, T. R. Flotte, A. MatsunoYagi, and T. Yagi, "In vivo complementation of complex I by the yeast Ndil enzyme: possible application for treatment of 
Parkinson disease," Journal of Biological Chemistry, vol. 281, no. 20, pp. 14250-14255, 2006.

[20] T. Yagi, B. B. Seo, E. Nakamaru-Ogiso et al., "Possibility of transkingdom gene therapy for complex I diseases," Biochimica et Biophysica Acta, vol. 1757, no. 5-6, pp. 708-714, 2006.

[21] C. V. Borlongan and P. R. Sanberg, "Elevated body swing test: a new behavioral parameter for rats with 6- hydroxydopamineinduced hemiparkinsonism," Journal of Neuroscience, vol. 15, no. 7, part 1, pp. 5372-5378, 1995.

[22] R. Iancu, P. Mohapel, P. Brundin, and G. Paul, "Behavioral characterization of a unilateral 6-OHDA-lesion model of Parkinson's disease in mice," Behavioural Brain Research, vol. 162, no. 1, pp. 1-10, 2005.

[23] M. Marella, B. B. Seo, E. Nakamaru-Ogiso, J. T. Greenamyre, A. Matsuno-Yagi, and T. Yagi, "Protection by the NDI1 gene against neurodegeneration in a rotenone rat model of Parkinson's disease," PLos One, vol. 3, no. 1, Article ID e1433, 2008.

[24] M. Marella, B. B. Seo, B. B. Thomas, A.. Matsuno-Yagi, and T. Yagi, "Successful amelioration of mitochondrial optic neuropathy using the yeast NDI1 gene in a rat animal model," Plos One, vol. 5, no. 7, Article ID e11472, 2010.

[25] M. D. Abràmoff, P. J. Magalhães, and S. J. Ram, "Image processing with imageJ," Biophotonics International, vol. 11, no. 7, pp. 36-41, 2004.

[26] M. E. Emborg, M. Carbon, J. E. Holden et al., "Subthalamic glutamic acid decarboxylase gene therapy: changes in motor function and cortical metabolism," Journal of Cerebral Blood Flow and Metabolism, vol. 27, no. 3, pp. 501-509, 2007.

[27] D. N. Abrous, J. J. Rodriguez, M.-F. Montaron, C. Aurousseau, M. Le Moal, and P. Barneoud, "Behavioural recovery after unilateral lesion of the dopaminergic mesotelencephalic pathway: effect of repeated testing," Neuroscience, vol. 84, no. 1, pp. 213-221, 1998.

[28] C. J. Pycock, "Turning behaviour in animals," Neuroscience, vol. 5, no. 3, pp. 461-514, 1980.

[29] J. M. Henderson, S. Watson, G. M. Halliday, T. Heinemann, and M. Gerlach, "Relationships between various behavioural abnormalities and nigrostriatal dopamine depletion in the unilateral 6-OHDA-lesioned rat," Behavioural Brain Research, vol. 139, no. 1-2, pp. 105-113, 2003.

[30] E. Bezard, S. Dovero, B. Bioulac, and C. E. Gross, "Kinetics of nigral degeneration in a chronic model of MPTP-treated mice," Neuroscience Letters, vol. 234, no. 1, pp. 47-50, 1997.

[31] B. B. Seo, E. Nakamaru-Ogiso, T. R. Flotte, T. Yagi, and A. Matsuno-Yagi, "A single-subunit NADH-quinone oxidoreductase renders resistance to mammalian nerve cells against complex I inhibition," Molecular Therapy, vol. 6, no. 3, pp. 336-341, 2002.

[32] M. W. Jakowec, K. Nixon, E. Hogg, T. McNeill, and G. M. Petzinger, "Tyrosine hydroxylase and dopamine transporter expression following 1-methyl-4-phenyl-1,2,3,6tetrahydropyridine-induced neurodegeneration of the mouse nigrostriatal pathway," Journal of Neuroscience Research, vol. 76, no. 4, pp. 539-550, 2004.

[33] S. Przedborski and M. Vila, "The 1-methyl-4-phenyl-1,2,3,6tetrahydropyridine mouse model: a tool to explore the pathogenesis of Parkinson's disease," Annals of the New York Academy of Sciences, vol. 991, pp. 189-198, 2003.

[34] M. Gerlach, P. Riederer, H. Przuntek, and M. B. H. Youdim, "MPTP mechanisms of neurotoxicity and their implications for Parkinson's disease," European Journal of Pharmacology, vol. 208, no. 4, pp. 273-286, 1991.
[35] S. Przedborski, K. Tieu, C. Perier, and M. Vila, "MPTP as a mitochondrial neurotoxic model of Parkinson's disease," Journal of Bioenergetics and Biomembranes, vol. 36, no. 4, pp. 375-379, 2004.

[36] B. B. Seo, M. Marella, T. Yagi, and A. Matsuno-Yagi, "The single subunit $\mathrm{NADH}$ dehydrogenase reduces generation of reactive oxygen species from complex I," FEBS Letters, vol. 580, no. 26, pp. 6105-6108, 2006.

[37] M. Marella, B. B. Seo, A. Matsuno-Yagi, and T. Yagi, "Mechanism of cell death caused by complex I defects in a rat dopaminergic cell line," Journal of Biological Chemistry, vol. 282, no. 33, pp. 24146-24156, 2007. 


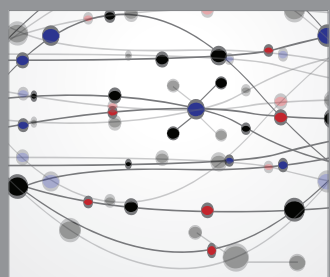

The Scientific World Journal
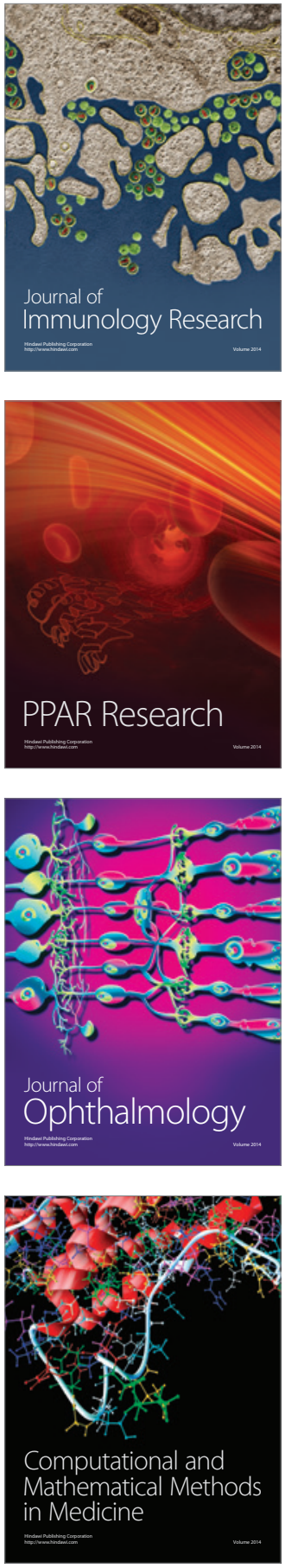

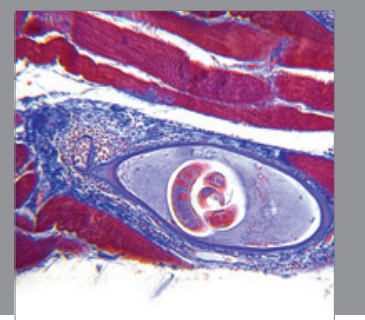

Gastroenterology

Research and Practice
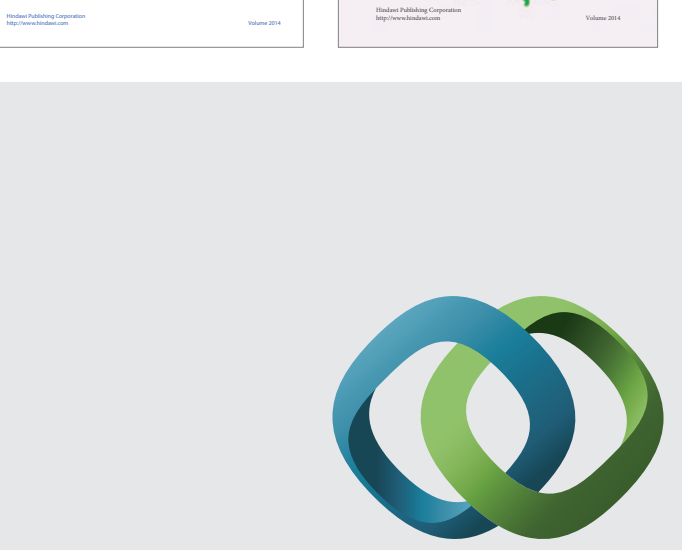

\section{Hindawi}

Submit your manuscripts at

http://www.hindawi.com
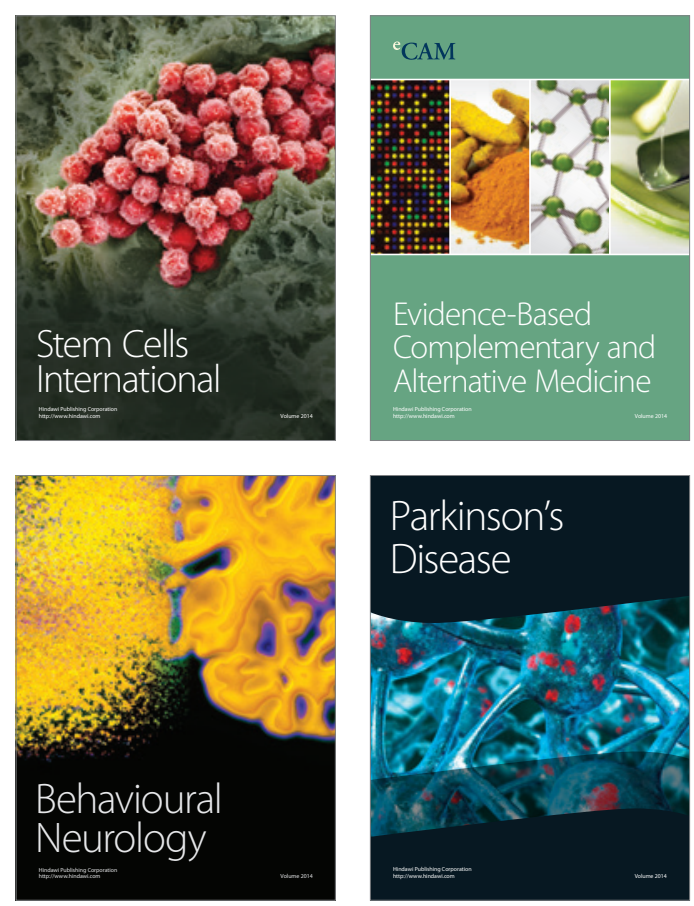

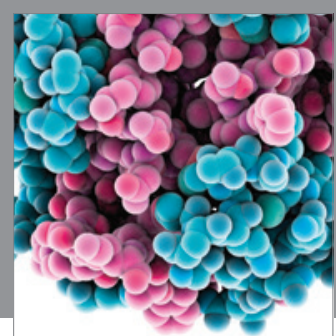

Journal of
Diabetes Research

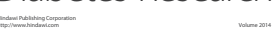

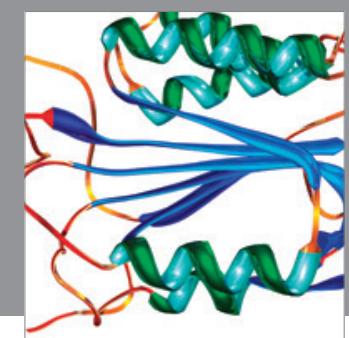

Disease Markers
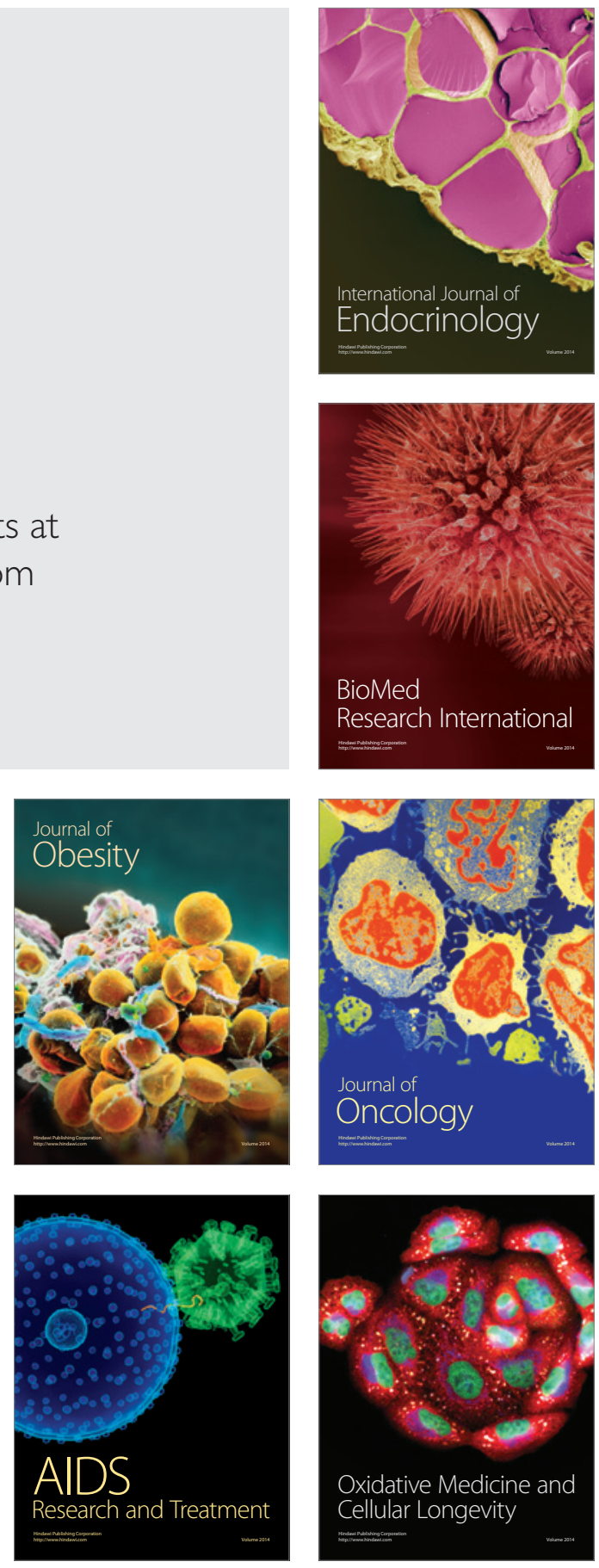